\title{
Gastrópodes (Mollusca) presentes no campus Urca da Universidade Federal do Estado do Rio de Janeiro (UNIRIO)
}

\author{
Guilherme de Lima Alexandre * \\ Hellen Virgínie Damasceno \\ Igor Christo Miyahira \\ Carlos Henrique Soares Caetano \\ Universidade Federal do Estado do Rio de Janeiro, Centro de Ciências Biológicas e da Saúde \\ Instituto de Biociências, Departamento de Zoologia \\ Avenida Pasteur, 458, CEP 22290-240, Rio de Janeiro - RJ, Brasil \\ * Autor para correspondência \\ guilherme_lima_alexandre@hotmail.com
}

Submetido em 10/05/2017

Aceito para publicação em 28/07/2017

\section{Resumo}

Gastropoda é o grupo mais diverso de Mollusca, contudo ainda existem muitas lacunas no conhecimento, e os moluscos terrestres e de água doce são um exemplo. Desta forma, apresentamos o levantamento dos gastrópodes do campus Urca da Universidade Federal do Estado do Rio de Janeiro (UNIRIO), área limítrofe com o Parque Natural Municipal da Paisagem Carioca e inclusa na área de amortecimento do Monumento Natural dos Morros do Pão de Açúcar e da Urca. Foram selecionados 11 pontos de amostragem e utilizamos uma metodologia direta de coleta. Ao todo foi encontrado um total de 1829 exemplares distribuídos em 18 espécies dentro de 12 famílias. O presente estudo pode mostrar a importância de áreas urbanas protegidas na conservação da fauna, tendo em vista o quantitativo de espécies nativas encontradas e o registro de primeira ocorrência de uma das espécies para o estado do Rio de Janeiro.

Palavras-chave: Biodiversidade; Fauna urbana; Levantamento faunístico

\section{Abstract}

Gastropods (Mollusca) present on the Urca campus of the Universidade Federal do Estado do Rio de Janeiro (UNIRIO). Gastropoda is the most diverse group of Mollusca. However, many gaps still exist in the knowledge of this group, for example, for terrestrial and freshwater gastropods. Thus, this work presents a mollusk survey of the Urca campus of the Universidade Federal do Estado do Rio de Janeiro, which is bordered by Parque Natural Municipal da Paisagem Carioca and is within the buffer zone of Monumento Natural dos Morros do Pão de Açúcar e da Urca. Eleven collecting sites were chosen and the mollusks were collected directly. A total of 1,829 specimens, distributed in 18 species and 12 families, were found. Considering the number of native species and the first record of one species for Rio de Janeiro State, the present study shows the importance of protected urban areas in the conservation of fauna.

Key words: Biodiversity; Faunistic survey; Urban fauna 


\section{Introdução}

Os moluscos constituem um grupo monofilético, sendo o segundo maior filo em número de espécies, com aproximadamente 200 mil viventes, além de um vasto registro fóssil (PONDER; LINDBERG, 2008). Embora sua maior diversidade se encontre no ambiente marinho, os gastrópodes apresentaram linhagens evolutivas que conseguiram explorar de maneira bem sucedida os ambientes terrestres e de água doce (SIMONE, 2006).

Estudos envolvendo gastrópodes terrestres no Brasil são escassos, com grande parte dos dados disponíveis baseados em estudos antigos, sendo a morfologia e distribuição das espécies pouco conhecida (RODRIGUES et al., 2016).

Com o avanço do homem sobre a natureza, inúmeros habitat naturais foram destruídos, levando muitos animais a se adaptar a um ambiente urbano, surgindo assim a chamada "fauna urbana", onde os animais vivem em convívio com os humanos, usufruindo dos recursos oferecidos por esse ambiente, tornando assim as cidades verdadeiros redutos ecológicos (NUNES, 2011). O município do Rio de Janeiro é propício a essa interação, pois conserva, em seu interior, alguns fragmentos de Mata Atlântica, estando assim as áreas urbanas em contato com as florestas.

Apesar de trabalhos faunísticos em áreas urbanas serem muitas vezes considerados como de "pouca importância", essas áreas podem sustentar importantes amostras da nossa biodiversidade. Recentemente, houve a descrição de uma nova espécie de gastrópode terrestre a partir de material oriundo de um parque urbano de São Paulo (MARTINS; SIMONE, 2014).

O campus Urca da UNIRIO fica localizado no município do Rio de Janeiro, na região Metropolitana do estado do Rio de Janeiro, e apresenta um clima tropical atlântico com baixa amplitude térmica. Esse local encontra-se entre importantes unidades de conservação do município do Rio de Janeiro, estando incluído na zona de amortecimento do Monumento Natural dos Morros do Pão de Açúcar e da Urca, além de fazer limite com setor Babilônia do Parque Natural Municipal da Paisagem Carioca (SMAC, 2017).
Com o objetivo de conhecer as espécies de moluscos do campus Urca da Universidade Federal do Estado do Rio de Janeiro (UNIRIO), foi realizado um levantamento faunístico no local, englobando todos os ambientes possíveis da ocorrência de moluscos.

\section{Materiais e Métodos}

Foi realizada uma análise prévia da área do campus, na qual foram eleitos 11 pontos de coleta (Figura 1), descritos brevemente a seguir: Ponto 1 (P1) - Presença de serapilheira, vegetação rasteira, próximo à borda do morro da Babilônia (22 ${ }^{\circ} 57^{\prime} 19.55^{\prime}$ 'S - 4310’07.71”O). Ponto 2 (P2) - Presença de serapilheira, vegetação rasteira e mata em regeneração (2257'19.05”S - 4310’07.19”O). Ponto 3 (P3) Ambiente aquático artificial controlado com macrófitas

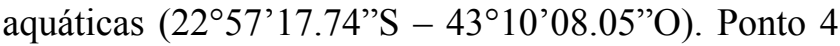
(P4) - Ambiente rochoso com presença de pontos com vegetação rasteira (2257'19.17’'S - 4310'07.53”O). Ponto 5 (P5) - Presença de serapilheira e com mata em regeneração (22॰57'19.04”S - 43¹0’08.49”O). Ponto 6 (P6) - Presença de serapilheira e com mata em regeneração, próximo à borda do morro da Babilônia

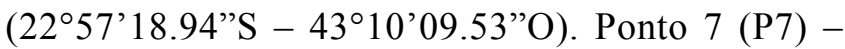
Presença de serapilheira e com mata em regeneração, próximo à borda do morro da Babilônia (2257'19.06”'S 4310'16.11”O). Ponto 8 (P8) - Presença de serapilheira e com mata em regeneração, próximo à borda do morro da Babilônia (22 $57^{\prime} 18.77^{\prime}$ 'S - 4310'16.15’O). Ponto 9 (P9) - Presença de serapilheira e vegetação rasteira com algumas árvores (2257'15.61'”S - 4310’09.37’O). Ponto 10 (P10) - Presença de serapilheira e vegetação rasteira com algumas árvores $\left(22^{\circ} 57^{\prime} 18.66\right.$ "S 4310'13.48”O). Ponto 11 (P11) - Presença de serapilheira e vegetação rasteira com algumas árvores (2257'18.02”S - 43¹0'12.54”O).

Cada um dos 11 pontos de coleta foi vistoriado três vezes, em três períodos de coleta distintos, o primeiro de março/2015 a abril/2015, o segundo de agosto/2015 a setembro/2015 e o terceiro de julho/2016 a agosto/2016. A busca dos moluscos foi realizada através de um método de coleta direta com o mesmo esforço amostral de duas pessoas/hora em cada um dos pontos. Após a coleta, 
FIGURA 1: Localização dos pontos de coleta no Campus Urca da Universidade Federal do Estado do Rio de Janeiro, no detalhe a localização do município do Rio de Janeiro dentro do estado do Rio de Janeiro. (Imagem original retirada do Google Earth).

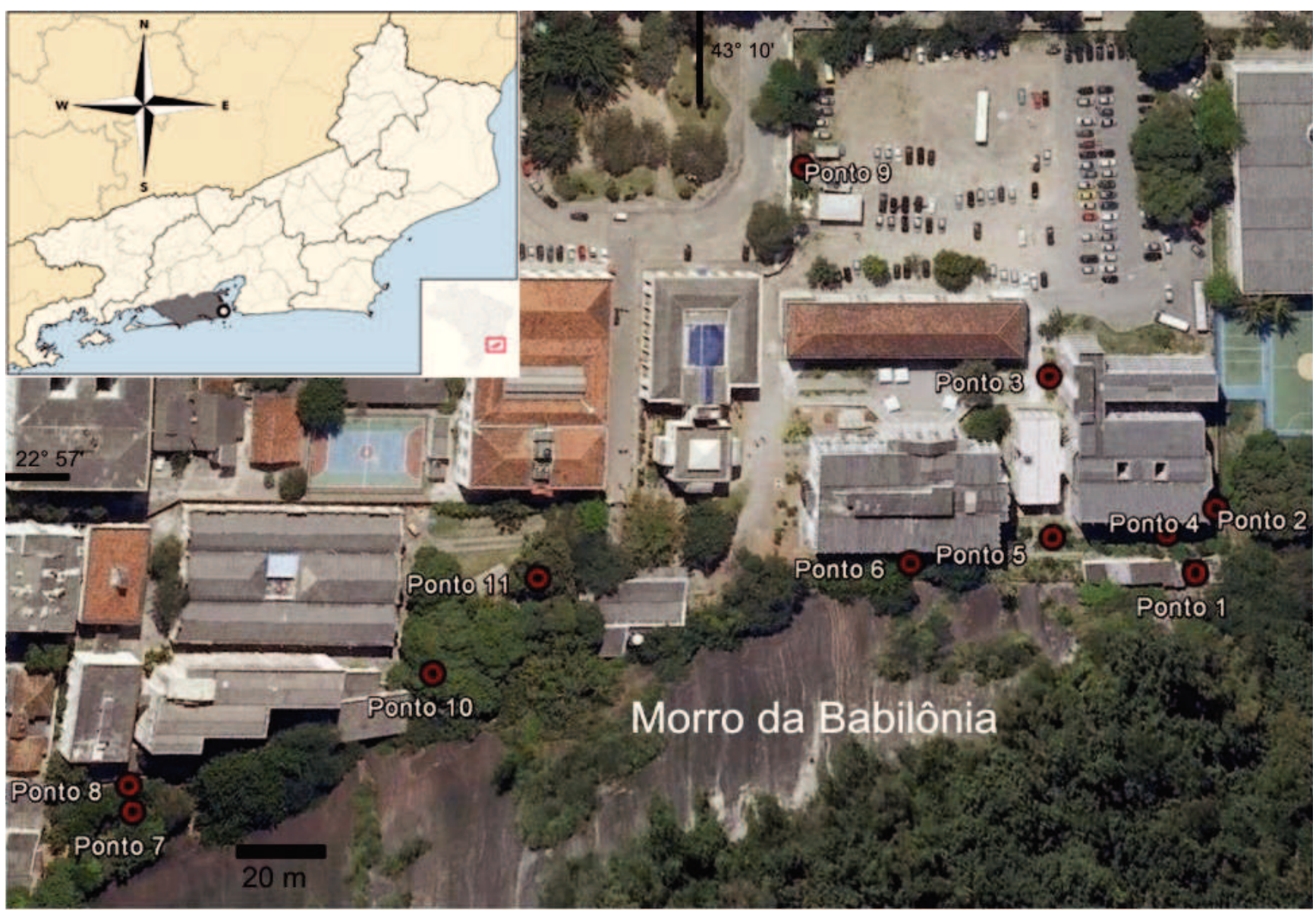

todos os indivíduos foram levados para o laboratório para quantificação e identificação.

Os espécimes terrestres foram relaxados imersos em água e os dulceaquícolas anestesiados com o auxílio de mentol. O tempo de relaxamento e anestesia não foi padronizado, visto que o tempo de reação era diferente para cada espécie. Posteriormente ambos foram conservados em álcool 70\%. As conchas vazias foram preservadas a seco.

Todos os indivíduos foram observados na lupa, quantificados e identificados com o auxílio de bibliografia (e.g., SIMONE, 2006; THOMÉ et al., 2006; MANSUR et al., 2012) e consulta a especialistas quando possível. A classificação seguiu Bouchet e Rocroi (2005). As fotos das conchas foram feitas com o auxílio de um estereomicroscópio (Olympus SZX12) com uma câmera fotográfica (Olympus DP70) acoplada.

As espécies encontradas foram classificadas em nativas e exóticas de acordo com a literatura pertinente (TRYON; PILSBRY, 1885; ANDREWS; DUNDEE, 1987; MONTEIRO; SANTOS, 2001; PARAENSE, 2001; MAURER et al., 2002; BRONSON; GASKALLA, 2003; BOGÉA et al., 2005; PHIRI et al., 2005; THIENGO et al., 2006; ARRUDA et al., 2007; SILVA, 2007; ROBINSON et al., 2009; SUTCHARIT et al., 2010; LOPES et al., 2012; PILATE et al., 2012; OLIVEIRA et al., 2015; BENSON, 2016) e depois comparada com outros estudos similares.

Material testemunho foi depositado na coleção malacológica do Museu Nacional da Universidade Federal do Rio de Janeiro (MNRJ). 


\section{Resultados}

Foi coletado um total de 1829 exemplares distribuídos em 18 espécies dentro de 12 famílias (Tabela 1; Figura 2), são elas: Melanoides tuberculata (Müller, 1774) (Thiaridae); Lymnaea collumela (Say, 1817) (Lymnaeidae); Biomphalaria glabrata (Say, 1818) (Planorbidae); Physa acuta (Draparnaud, 1805) (Physidae); Sarasinula linguaeformis (Semper, 1885) (Veronicellidae); Omalonyx matheroni (Potiez \& Michaud, 1838) (Succinaidae); Bulimulus tenuissimus (d'Orbigny, 1835) (Orthalicidae); Rhinus cf. ciliatus (Gould, 1846) (Orthalicidae); Lamellaxis (Leptopeas) mizius Marcus \& Marcus, 1968 (Subulinidae); Dysopeas muibum Marcus \& Marcus, 1968 (Subulinidae); Leptinaria unilamellata (d'Orbigny, 1835) (Subulinidae); Beckianum beckianum (Pfeifer, 1846) (Subulinidae); Subulina octona (Bruguière, 1792) (Subulinidae); Streptaxis contusus (Férussac, 1821) (Streptaxidae); Streptartemon cookeanus (Baker, 1914) (Streptaxidae); Tamayoa banghaasi (Boettger in Thiele, 1927) (Scolodontidae); Pseudoguppya semenlini (Moricand, 1846) (Euconulidae) e Bradybaena similaris (Férussac, 1821) (Bradybaenidae). As famílias representadas por mais espécies foram Subulinidae $(\mathrm{n}=5)$, Streptaxidae $(\mathrm{n}=2)$ e Orthalicidae $(\mathrm{n}=2)$, todas as demais famílias foram representadas por apenas uma espécie (Figura 3).
Do total de 18 espécies, 13 espécies foram encontradas em todas as coletas. Streptaxis contusus só ocorreu na primeira coleta, Rhinus cf. ciliatus e Lamellaxis (Leptopeas) mizius na segunda e não houve espécies exclusivas na terceira. As espécies com maior distribuição pelo campus foram Subulina octona e Streptartemon cookeanus, ocorrendo em 10 pontos de coleta. As espécies mais abundantes foram Subulina octona $(\mathrm{n}=1326)$ e Streptartemon cookeanus $(\mathrm{n}=162)$, as menos abundantes foram Rhinus cf. ciliatus $(\mathrm{n}=1)$, Lamellaxis (Leptopeas) mizius $(\mathrm{n}=1)$ e Streptaxis contusus $(\mathrm{n}=1)$. Os pontos com maior número de espécies foram o P5 $(\mathrm{n}=8)$ seguido por P1 e P11 (n=6), e o ponto com menor número de espécies $(n=3)$ foi o Pl0 (Figura 4). As espécies que apresentaram distribuição mais restrita foram Melanoides tuberculata, Lymnaea columella, Biomphalaria glabrata, Physa acuta e Omalonyx matheroni, que ocorreram em apenas um ponto de coleta (P3), o único que possuía um ambiente aquático. Entre as espécies terrestres, as de menor distribuição no campus foram Rhinus cf. ciliatus, Lamellaxis (Leptopeas) mizius e Streptaxis contusus, ocorrendo somente em um dos pontos de coleta. 
TABELA 1: Total de indivíduos coletados com os respectivos táxons e ocorrência global.

\begin{tabular}{|c|c|c|c|c|}
\hline Clado & Família & Espécie & Ocorrência Unirio $* * *$ & Ocorrência global \\
\hline Sorbeoconcha & $\begin{array}{l}\text { Thiaridae Gill, } \\
1871\end{array}$ & $\begin{array}{l}\text { Melanoides tuberculata } \\
\text { (Müller, 1774) }\end{array}$ & $\mathrm{P} 3(15)$ & $\begin{array}{l}\text { Brasil*, Europa, Leste } \\
\text { asiático, Norte e Leste da } \\
\text { África }\end{array}$ \\
\hline \multirow[t]{3}{*}{ Hygrophila } & $\begin{array}{l}\text { Lymnaeidae } \\
\text { Rafinesque, } 1815\end{array}$ & $\begin{array}{l}\text { Lymnaea columella (Say, } \\
\text { 1817) }\end{array}$ & $\mathrm{P} 3(6)$ & $\begin{array}{l}\text { América do Norte, } \\
\text { América Central, América } \\
\text { do Sul*, Europa }\end{array}$ \\
\hline & $\begin{array}{l}\text { Planorbidae } \\
\text { Rafinesque, } 1815\end{array}$ & $\begin{array}{l}\text { Biomphalaria glabrata } \\
\text { (Say, 1818) }\end{array}$ & P3(13) & $\begin{array}{l}\text { América Central, América } \\
\text { do Sul** }\end{array}$ \\
\hline & $\begin{array}{l}\text { Physidae } \\
\text { Fitzinger, } 1833\end{array}$ & $\begin{array}{l}\text { Physa acuta Draparnaud, } \\
1805\end{array}$ & $\mathrm{P} 3(22)$ & $\begin{array}{l}\text { Brasil*, Europa, } \\
\text { Mediterrâneo, África, } \\
\text { América Central }\end{array}$ \\
\hline Systellommatophora & $\begin{array}{l}\text { Veronicellidae } \\
\text { Gray, } 1840\end{array}$ & $\begin{array}{l}\text { Sarasinula linguaeformis } \\
\text { (Semper, 1885) }\end{array}$ & $\mathrm{P} 8(5)$ & Brasil** \\
\hline \multirow[t]{13}{*}{ Stylommatophora } & $\begin{array}{l}\text { Succinaidae } \\
\text { Beck, } 1837\end{array}$ & $\begin{array}{l}\text { Omalonyx matheroni } \\
\text { (Potiez \& Michaud, 1835) }\end{array}$ & P3(6) & $\begin{array}{l}\text { Ilhas do Caribe, América } \\
\text { Central, América do Sul }{ }^{* *}\end{array}$ \\
\hline & $\begin{array}{l}\text { Orthalicidae } \\
\text { Albers, } 1860\end{array}$ & $\begin{array}{l}\text { Bulimulus tenuissimus } \\
\text { (d'Orbigny, 1835) }\end{array}$ & P2(1) P5(2) P9(1) & $\begin{array}{l}\text { Brasil**, América do } \\
\text { Norte }\end{array}$ \\
\hline & & $\begin{array}{l}\text { Rhinus cf. ciliatus (Gould, } \\
\text { 1846) }\end{array}$ & $\mathrm{P} 11(1)$ & Brasil, Venezuela \\
\hline & $\begin{array}{l}\text { Subulinidae P. } \\
\text { Fischer \&Crosse, } \\
1877\end{array}$ & $\begin{array}{l}\text { Lamellaxis (Leptopeas) } \\
\text { mizius Marcus \& Marcus, } \\
1968\end{array}$ & $\mathrm{P} 5(1)$ & $\begin{array}{l}\text { América do Norte, } \\
\text { América Central, América } \\
\text { do Sul** }\end{array}$ \\
\hline & & $\begin{array}{l}\text { Dysopeas muibum Marcus } \\
\text { \& Marcus, } 1968\end{array}$ & $\mathrm{P} 7(1)$ & Brasil** \\
\hline & & $\begin{array}{l}\text { Leptinaria unilamellata } \\
\text { (d'Orbigny, 1835) }\end{array}$ & P11(13) & Brasil*, Bolívia \\
\hline & & $\begin{array}{l}\text { Beckianum beckianum } \\
\text { (Pfeifer, 1846) }\end{array}$ & P1(6) P2(9) & $\begin{array}{l}\text { Brasil*, Bacia do Caribe, } \\
\text { América do Norte }\end{array}$ \\
\hline & & $\begin{array}{l}\text { Subulina octona } \\
\text { (Bruguière, 1792) }\end{array}$ & $\begin{array}{l}\text { P1(71) P4(4) P5(62) } \\
\text { P6(137) P7(214) P8(89) } \\
\text { P9(3) P10(90) P11(64) }\end{array}$ & $\begin{array}{l}\text { Brasil*, Cuba, Caribe, } \\
\text { Venezuela, África }\end{array}$ \\
\hline & \multirow[t]{2}{*}{$\begin{array}{l}\text { Streptaxidae } \\
\text { Gray, } 1860\end{array}$} & $\begin{array}{l}\text { Streptaxis contusus } \\
\text { (Férussac, 1821) }\end{array}$ & P5(1) P7(5) & Brasil** \\
\hline & & $\begin{array}{l}\text { Streptartemon cookeanus } \\
\text { (Baker, 1914) }\end{array}$ & $\begin{array}{l}\text { P1(9) P2(4) P4(7) P5(17) } \\
\text { P6(28) P7(5) P8(23) } \\
\text { P9(1) P10(4) P11(3) }\end{array}$ & Brasil** \\
\hline & $\begin{array}{l}\text { Scolodontidae H. } \\
\text { B. Baker, } 1925\end{array}$ & $\begin{array}{l}\text { Tamayoa banghaasi } \\
\text { (Boettger in Thiele, 1927) }\end{array}$ & $\begin{array}{l}\mathrm{P} 1(27) \mathrm{P} 2(5) \mathrm{P} 4(4) \mathrm{P} 5(9) \\
\mathrm{P} 7(7) \mathrm{P} 8(2) \mathrm{P} 10(8) \mathrm{P} 11(5)\end{array}$ & Brasil**, Paraguai \\
\hline & $\begin{array}{l}\text { Euconulidae } \\
\text { Baker, } 1928\end{array}$ & $\begin{array}{l}\text { Pseudoguppya semenlini } \\
\text { (Moricand, 1846) }\end{array}$ & P1(1) P4(1) & America do Sul** \\
\hline & $\begin{array}{l}\text { Bradybaenidae } \\
\text { Pilsbry, } 1934\end{array}$ & $\begin{array}{l}\text { Bradybaena similaris } \\
\text { (Férussac, 1821) }\end{array}$ & P5(4) P6(2) P9(7) & Brasil*, Leste asiático \\
\hline
\end{tabular}

* Espécies exóticas no Brasil. ** Espécies nativas do Brasil. *** Se refere aos pontos assinalados na Figura l, e os números entre parênteses se referem à quantidade de indivíduos coletados em cada ponto no somatório das três amostragens. 
FIGURA 2: Exemplares dos moluscos coletados na UNIRIO campus Urca; A - Melanoides tuberculatus (Müller, 1774); B - Lymnaea columella (Say, 1817); C - Biomphalaria glabrata (Say, 1818); D - Physa acuta (Draparnaud, 1805); E - Sarasinula linguaeformis (Semper, 1885); F - Omalonyx matheroni (Potiez \& Michaud, 1835); G - Bulimulus tenuissimus (d'Orbigny, 1835); H - Rhinus cf. ciliatus (Gould, 1846); I - Lamellaxis (Leptopeas) mizius; J - Dysopeas muibum (Marcus \& Marcus, 1968); K - Leptinaria unilamellata (d'Orbigny, 1835); L - Beckianum beckianum (Pfeifer, 1846); M - Subulina octona (Brugüière, 1789); N-Pseudoguppya semenlini (Moricand, 1846); O - Streptaxis contusus (Férussac, 182 1); P-Streptartemon cookeanus (Baker, 1914); Q - Tamayoa banghaasi (Boettger in Thiele, 1927); R - Bradybaena similaris (Férussac, 1821). Escala: B, C, E, G, M, O e R: 10 mm; A, D, F, H, K, L e P: 5 mm; I, J, N e Q: 1 mm.

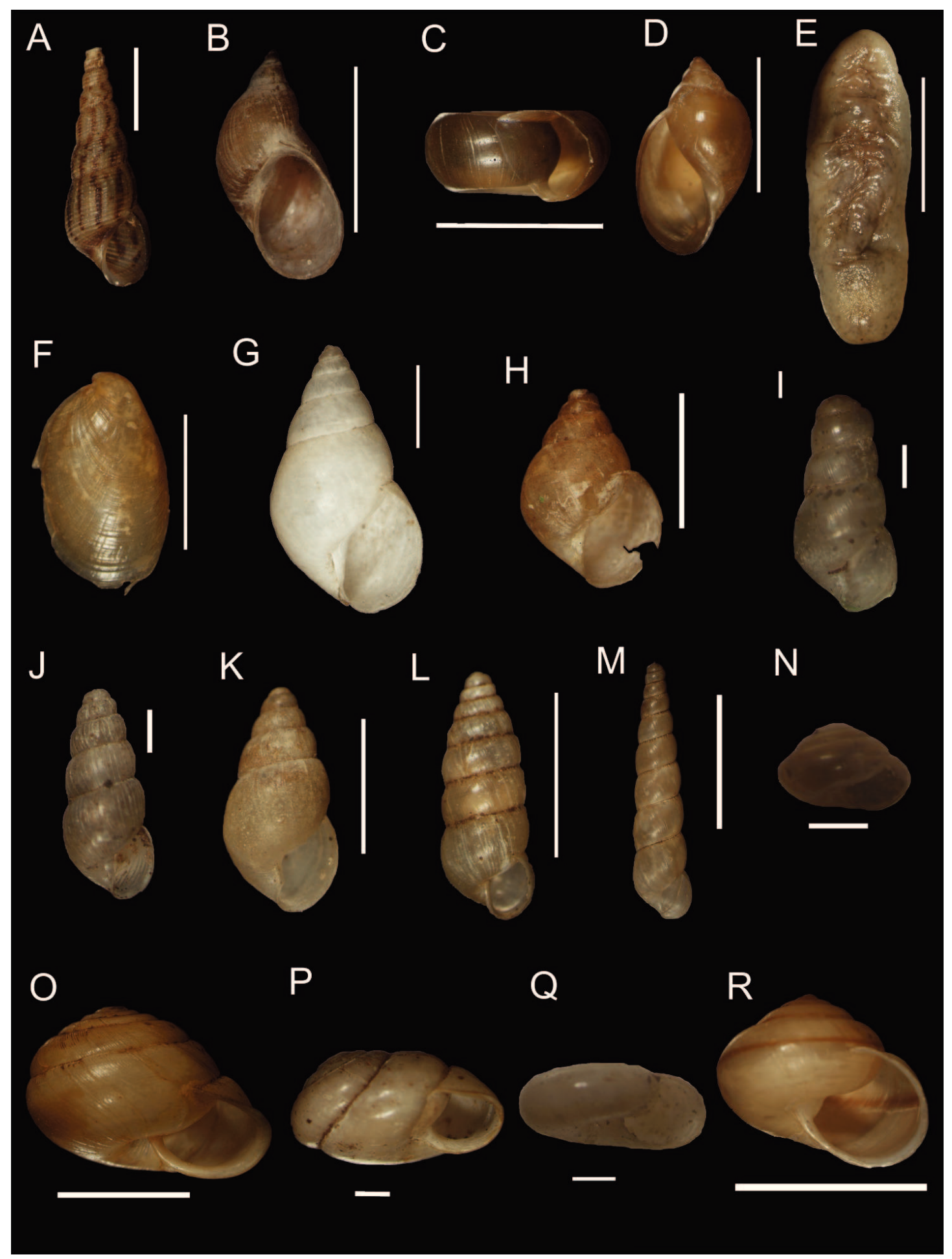


FIGURA 3: Número de espécies de moluscos encontradas em cada família no campus Urca da UNIRIO.

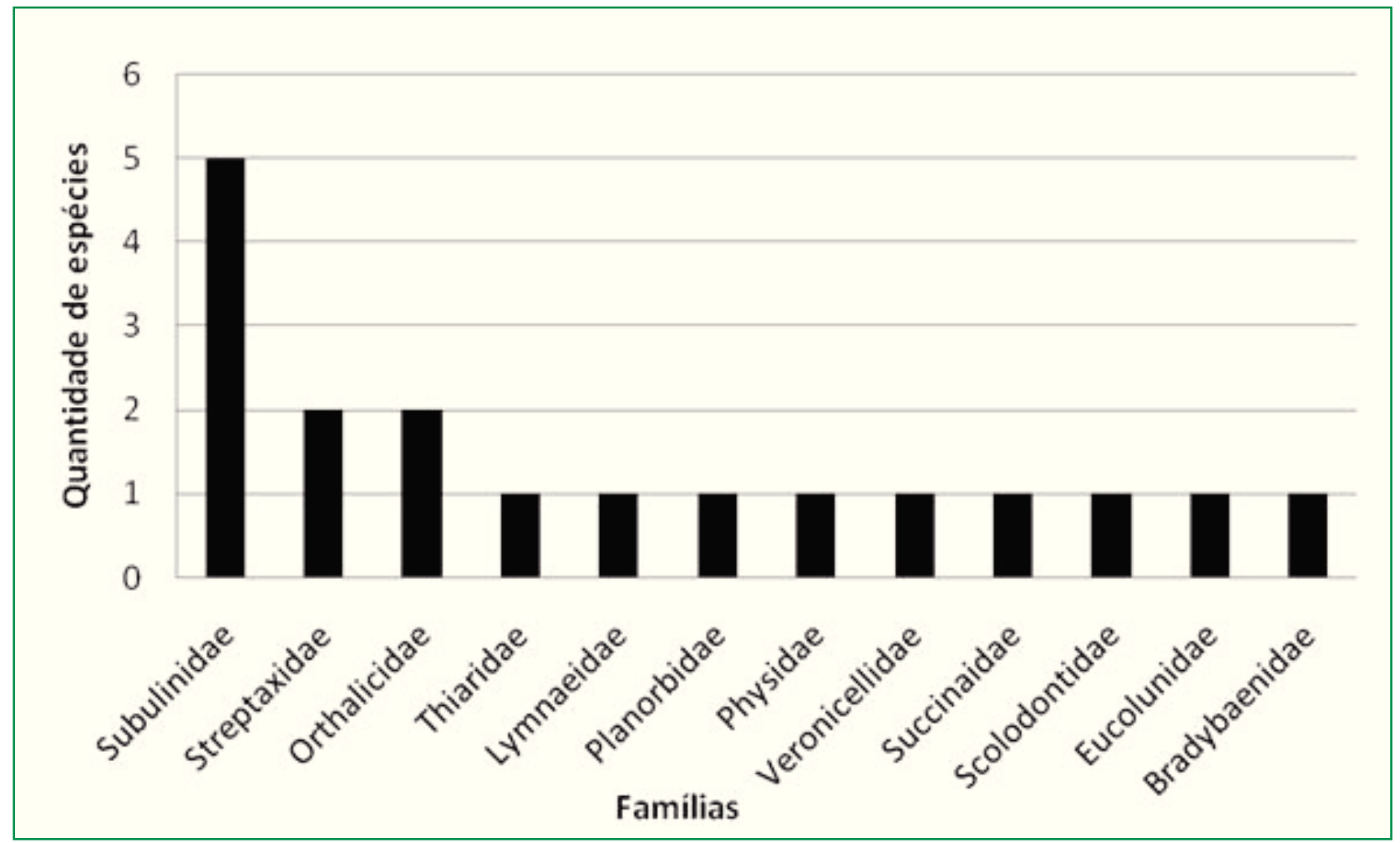

FIGURA 4: Quantidade de espécies coletadas por ponto de amostragem no campus Urca da Universidade Federal do Estado do Rio de Janeiro.

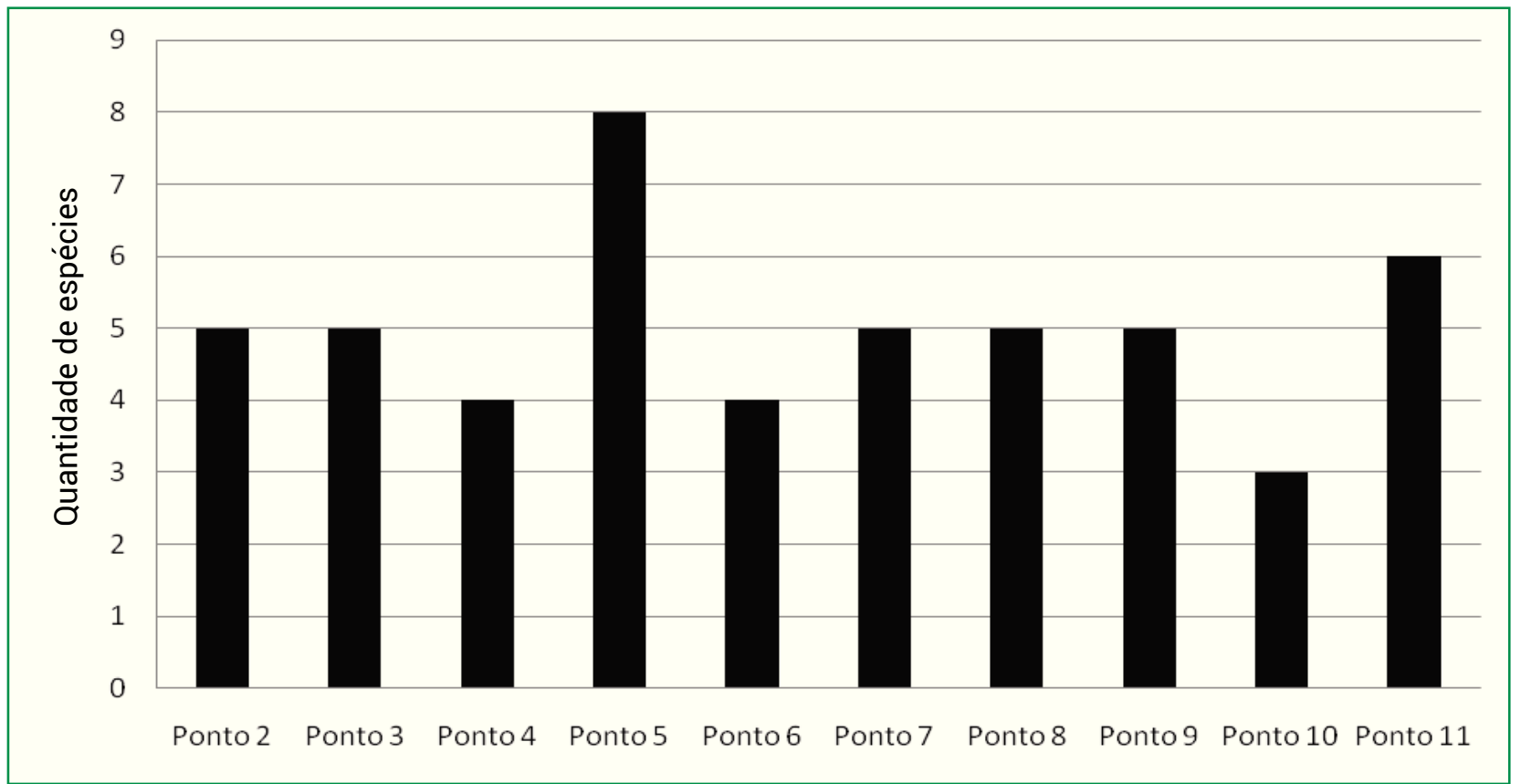


Entre as espécies encontradas, 11 são espécies nativas do Brasil, sete são espécies introduzidas e uma não foi possível determinar devido à identificação imprecisa. Em contrapartida, as abundâncias não seguiram esse padrão. A abundância total de exóticas obtidas nas três coletas foi quase quatro vezes maior que a abundância de espécies nativas (1472 exóticas e 354 nativas). Dysopeas muibum é registrado pela primeira vez para o estado do Rio de Janeiro.

\section{Discussão}

Assim como nos trabalhos de Santos (2001) e Lopes et al. (2012), Subulinidae foi a família mais abundante, sendo uma das espécies que ocorreram em um maior número de pontos (dez), Subulina octona, pertencente a esta família. Esta ampla distribuição e a grande abundância provavelmente são reflexos dos hábitos generalistas da espécie (BESSA; ARAÚJO, 1995). Esse fato também pode ser visto através da ampla distribuição mundial desta espécie (Tabela 1). Entre as espécies pouco abundantes e restritas à UNIRIO, foram obtidas tanto espécies nativas (Biomphalaria glabrata,
Omalonyx matheroni, Streptaxis contusus, Dysopeas muibum, Lamellaxis (Leptopeas) mizius, Pseudoguppya semenlini e Lymnaea columella) quanto espécies exóticas (Lymnaea columella, Physa acuta, Melanoides tuberculata e Beckianum beckianum). A espécie Rhinus cf. ciliatus não foi identificada a nível específico, pois foi obtido apenas um exemplar que estava danificado. Apesar de possuir número de voltas, abertura da concha e forma da concha condizentes com Rhinus ciliatus, não foram observadas projeções periostracais, que são características dessa espécie (BREURE; ESKENS, 1981).

Trabalhos recentes de levantamentos faunísticos em áreas antropizadas encontraram número menor de espécies que o apresentado neste trabalho, apesar de a área aqui estudada ser relativamente pequena. Rodrigues et al. (2016), em um levantamento prévio de moluscos terrestres em áreas urbanas de 16 municípios do estado do Rio de Janeiro, encontraram nove espécies distribuídas em sete famílias. Lopes et al. (2012) identificaram dez espécies em cinco famílias no campus Madureira do Centro Universitário da Cidade (UniverCidade), também no Rio de Janeiro (Tabela 2).

TABELA 2: Comparação entre espécies exóticas e nativas entre o atual estudo e os estudos de Lopes et al. (2012) e Rodrigues et al. (2016).

\begin{tabular}{|c|c|c|c|}
\hline Estudo & Espécies exóticas & Espécies nativas & Não determinadas \\
\hline $\begin{array}{l}\text { Presente estudo } \\
\text { (Campus Urca UNIRIO) }\end{array}$ & $\begin{array}{l}\text { Melanoides tuberculatus } \\
\text { Physa acuta } \\
\text { Leptinaria unilamellata } \\
\text { Beckianum beckianum } \\
\text { Subulina octona } \\
\text { Bradybaena similaris }\end{array}$ & $\begin{array}{l}\text { Lymnaea columella } \\
\text { Biomphalaria glabrata } \\
\text { Sarasinula linguaeformis } \\
\text { Omalonyx matheroni } \\
\text { Bulimulus tenuissimus } \\
\text { Lamellaxis (Lepetopeas) mizius } \\
\text { Dysopeas muibum } \\
\text { Streptaxis contusus } \\
\text { Streptartemon cookeanus } \\
\text { Tamayoa banghaasi } \\
\text { Pseudoguppya semenlini }\end{array}$ & Rhinus cf. ciliatus \\
\hline Lopes et al. (2012) & $\begin{array}{l}\text { Subulina octona } \\
\text { Leptinaria unilamellata } \\
\text { Opeas pyrgula } \\
\text { Bradybaena similaris }\end{array}$ & $\begin{array}{l}\text { Allopeas micra } \\
\text { Bulimulus tenuissimus } \\
\text { Streptartemon cookeanus } \\
\text { Huttonella bicolor }\end{array}$ & Scolodonta sp. \\
\hline Rodrigues et al. (2016) & $\begin{array}{l}\text { Achatina fulica } \\
\text { Deroceras laeve } \\
\text { Bradybaena similaris } \\
\text { Subulina octona } \\
\text { Leptinaria unilamellata }\end{array}$ & $\begin{array}{l}\text { Sarasinula linguaeformis } \\
\text { Sarasinula plebeia } \\
\text { Bulimulus tenuissimus } \\
\text { Streptartemon cookeanus }\end{array}$ & \\
\hline
\end{tabular}


A proporção entre as espécies de gastrópodes nativas e exóticas foi diferente entre os dois trabalhos comparados. Rodrigues et al. (2016) indicaram uma maior proporção nas espécies exóticas, representando $56 \%$ das espécies encontradas em seu estudo, enquanto Lopes et al. (2012) observaram 50\% de espécies nativas, $37 \%$ introduzidas e $13 \%$ não determinadas. No Campus Urca da UNIRIO, $61 \%$ eram nativas, $33 \%$ introduzidas e $6 \%$ não determinadas, proporção muito parecida com o trabalho de Lopes et al. (2012), em que as proporções foram de $50 \%$ nativas, $37 \%$ exóticas e $13 \%$ não determinadas. Contudo, esses trabalhos apresentaram metodologias diferenciadas de coleta, porém acreditamos que a comparação geral é válida apesar das diferenças metodológicas. Cabe também ressaltar que o presente estudo englobou espécies de moluscos dulciaquícolas, grupo não envolvido nesses dois outros trabalhos. Fernandez et al. (2001) encontraram oito espécies de moluscos de água doce do campus Manguinhos da Fundação do Instituto Oswaldo Cruz. Nesse trabalho, foram investigadas dezoito coleções hídricas, das quais treze apresentaram moluscos, o que provavelmente explica o maior número de espécies de água doce quando comparado com o presente estudo.

Achatina fulica Férussac 1821, apesar de amplamente distribuída no Brasil (THIENGO et al., 2006), não foi encontrada na área estudada. Somando-se a isso, foi obtido um número considerável de espécies nativas, ressaltando a importância da área na conservação das espécies e no abrigo de fauna nativa, características consideradas importantes tendo em vista que essa área está incluída na zona de amortecimento do Monumento Natural dos Morros do Pão de Açúcar e da Urca e é limítrofe com o Parque Natural Municipal da Paisagem Carioca.

Além disso, devido ao estudo ter sido realizado dentro de uma área universitária com fácil acesso dos alunos, esse estudo pode ser usado com fins didáticos, visando ao treinamento dos alunos, podendo ser abordados aspectos da coleta, curadoria, ecologia e morfologia.

Desta forma, apesar de o estudo ter sido realizado em uma área antropizada foi possível contribuir para a compreensão da distribuição das espécies no estado, inclusive com novos registros para o estado do Rio de Janeiro.

\section{Agradecimentos}

Agradecemos aos dois revisores anônimos, pelas contribuições realizadas no manuscrito; ao Dr. Amilcar Brum Barbosa e à Dra. Suzete Rodrigues Gomes, pelo auxílio na identificação dos espécimes; à UNIRIO, pelas bolsas concedidas, a GLA e a HVD, e pela estrutura fornecida.

\section{Referências}

ANDREWS, K. L.; DUNDEE, D. Las babosas Veronicellidos de Centroamerica con énfasis en Sarasinula plebeia (= Vaginuluspleheius). Ceiba, Tegucigalpa, v. 28, n. 2, p. 163-172, 1987.

ARRUDA, J. O.; GOMES, S. R.; RAMÍREZ, R.; THOMÉ, J. W. Morfoanatomia de duas espécies do gênero Omalonyx (Mollusca, Pulmonata, Succineidae) com novo registro para Minas Gerais, Brasil. Biociências, Porto Alegre, v. 14, n. 1, p. 61-70, 2007.

BENSON, A. Physella acuta. 2016. Disponível em: <http://nas. er.usgs.gov/queries/factsheet.aspx?SpeciesID $=1025>$. Acesso em: 19 abr. 2017.

BESSA, E. C. A.; ARAÚJO, J. L. B. Ocorrência de autofecundação em Subulina octona (Bruguiére) (Pulmonata, Subulinidae) sob condições de laboratório. Revista Brasileira de Zoologia, Curitiba, v. 12, n. 3, p 719-723, 1995.

BOG A, T.; CORDEIRO, F. M.; GOUVEIA, J. S. Melanoides tuberculatus (Gastropoda: Thiaridae) as intermediate host of Heterophyidae (Trematoda: Digenea) in Rio de Janeiro metropolitan area, Brazil. Revista do Instituto de Medicina tropical de São Paulo, São Paulo, v. 47, n. 2, p. 87-90, 2005.

BOUCHET, P.; ROCROI, J. P. Classification and Nomenclator of Gastropod Families, Malacologia, Hackenheim, v. 47, n. 1-2, p. 1-397, 2005.

BREURE, A. S. H.; ESKENS, A. A. C. Notes on and descriptions of Bulimulidae (Mollusca, Gastropoda), II. Zoologische Verhandelingen, Leiden, v. 186, n. 1, p. 1-111, 1981.

BRONSON, C.; GASKALLA, R. Invasive mollusk survey of Miami-Dade and Broward Counties. 2003. Disponível em: <https:/www.freshfromflorida.com/content/ download/9859/135422/pdf invasive_mollusk_survey_june2003. pdf > . Acesso: 19 abr. 2017.

FERNANDEZ, M. A.; THIENGO, S. C.; BOAVENTURA M. F. Gastrópodes límnicos do Campus de Manguinhos, Fundação Oswaldo Cruz, Rio de Janeiro, RJ. Revista da Sociedade Brasileira de Medicina Tropical, Uberaba, v. 34, n. 3, p. 279-282, 2001.

LOPES, M. P. A. M.; NUNES, G. K. M.; DOS SANTOS, S. B. Levantamento preliminar da malacofauna do Campus do Centro Universitário da Cidade (Univercidade, Unidade Madureira), Rio 
de Janeiro, RJ. Disponível em: <http://sbmalacologia.com.br/wpcontent/uploads/2011/11/Informativo-43-182.pdf>.

MANSUR, M. C. D.; SANTOS, C. P.; PEREIRA, D.; PAZ, I. C. P.; ZURITA, M. L. T.; RODRIGUEZ, M. T. R.; NEHRKE, M. V.; BERGONCI, P. E. A. Moluscos límnicos invasores do Brasil. Porto Alegre: Redes Editora, 2012. 411 p.

MARTINS, C. M.; SIMONE R. L. Anew species of Adelopoma from São Paulo urban park, Brazil (Caenogastropoda, Diplommatinidae). Journal of Conchology, Shefford, v. 41, n. 6, p. 765-773, 2014.

MAURER, R. L.; GRAEFF-TEIXEIRA, C.; THOM , J. W.; CHIARADIA, L. A.; SUGAYA, H.; YOSHIMURA, K. Natural infection of Deroceras laeve (Mollusca: Gastropoda) with Metastrongylid larvae in a transmission focus of abdominal Angiostrongyliasis. Revista do Instituto de Medicina Tropical de São Paulo, São Paulo, v. 44, n. 1, p. 53-54, 2002.

MONTEIRO, D. P.; SANTOS, S. B. Conquiliomorfologia de Tamayoa (Tamayops) banghaasi (Thiele) (Gastropoda, Systrophiidae). Revista Brasileira de Zoologia, Curitiba, v. 18, n. 4, p. 1049-1055, 2001.

NUNES, M. Fauna urbana - A vida selvagem à nossa porta. 2011. Disponível em: < https://cultivarbiodiversidade.wordpress. com/2011/09/10/fauna-urbana-\%E2\%80\%93-a-vida-selvagem-anossa-porta/>. Acesso em: 22 mar. 2016.

OLIVEIRA, A. P. M.; GENTILE, R.; JÚNIOR, A. M.; TORRES, E. J. L.; THIENGO, S. C. Angiostrongylus cantonensis infection in molluscs in the municipality of São Gonçalo, a metropolitan area of Rio de Janeiro, Brazil: role of the invasive species Achatina fulica in parasite transmission dynamics. Memories Instituto Oswaldo Cruz, Rio de Janeiro, v. 110, n. 6, p. 739-744, 2015.

PARAENSE, W. L. The schistosome vectors in the Americas. Memories Instituto Oswaldo Cruz, Rio de Janeiro, v. 96, n. 1, p. 7-16, 2001.

PHIRI, A. M.; PHIRI, I. K.; SIKASUNGE, C. S.; MONRAD, J. Prevalence of fasciolosis in Zambian cattle observed at selected abattoirs with emphasis on age, sex and origin. Journal of Veterinary Medicine, Cairo, v. 52, n. 9, p. 414-416, 2005.

PILATE, J. V.; SILVA, L. C.; VARGAS, T.; SOUZA, B. A.; DURÇO, E. C.; BESSA, E. C. A. Repertório comportamental e horário de atividade do molusco terrestre Dysopeas muibum MARCUS \& MARCUS, 1968 (Mollusca, Subulinidae) em laboratório. Revista de Biologia e Farmácia, Campina Grande, v. 8, n. 2, p. 176-188, 2012.

PONDER, W. F.; LINDBERG, D. R; Phylogeny and evolution of the Mollusca. London: University of California Press, 2008. 469 p.
ROBINSON, D. G.; HOVESTADT, A.; FIELDS, A.; BREURE, A. $\mathrm{S}$. H. The land Mollusca of Dominica (Lesser Antilles), with notes on some enigmatic or rare species. Zoologische Mededelingen, Leiden, v. 83, n. 13, p. 615-650, 2009.

RODRIGUES, P.; FERNANDEZ M. A.; THIENGO, S. C.; SALGADO, N. C.; GOMES, S. R. Diversity of terrestrial molluses in urban areas and surrounding land scapes of Rio de Janeiro State, Brasil. Tentacle, Hawaii, v. 24, n. 1, p. 39-41, 2016.

SANTOS, S. B.; MONTEIRO, P. D. Composição de gastrópodes terrestres em duas áreas do Centro de Estudos Ambientais e Desenvolvimento Sustentado (CEADS), Vila Dois Rios, Ilha Grande, Rio de Janeiro, Brasil - um estudo-piloto. Revista brasileira de Zoologia, Curitiba, v. 18, n. 1, p. 181-190, 2001.

SILVA, A. L. Habroconus (Pseudogupya) semenlini (Moricand, 1845) (Gastropoda, Stylommatophora, Euconulidae) e malacofauna acompanhante de um fragmento de floresta nativa alterada, no Campus do Vale da Universidade Federal do Rio Grande do Sul, Porto Alegre, Brasil. 2007. 107 f. Dissertação (Mestrado em Biologia Animal) - Universidade Federal do Rio Grande do Sul, Porto Alegre. 2007.

SIMONE, L. R. L. Land and freshwater molluses of Brazil. São Paulo: FAPESP, 2006. 390 p.

SMAC - Secretaria Municipal de Meio Ambiente. Unidades de conservação sob tutela municipal. 2017. Disponível em: $<$ http:// www.rio.rj.gov.br/web/smac/exibeconteudo?id=5590726>. Acesso em: 16 nov. 2017.

SUTCHARIT, C.; NAGGS, F.; WADE, M. C.; FONTANILLA, I.; PANHA, S. The new family Diapheridae, a new species of Diaphera Albers from Thailand, and the position of the Diapheridae within a molecular phylogeny of the Streptaxoidea (Pulmonata: Stylommatophora). Zoological Journal of the Linnean Society, London, v. 160, n. 1, p. 1-16, 2010.

THIENGO, S. C.; FARACO, F. A.; SALGADO, N. C.; COWIE, R. H.; FERNANDEZ, M. A. Rapid spread of an invasive snail in South America: the giant African snail, Achatina fulica, in Brazil, Biological Invasions, Dordrecht, v. 9, n. 6, p. 693-702, 2006.

THOM , J. W.; PICAN O, J. B.; GOMES, S. R. Os caracóis e lesmas dos nossos bosques e jardins. Pelotas: USEB, 2006. 123 p. TRYON, G. W.; PILSBRY, H. A. Manual of conchologist, structural and systematic. With illustrations of the species. Philadelphia: Academy of Natural Sciences of Philadelphia, 1885. $262 \mathrm{p}$. 